\title{
Plataforma para Análise de Dados de Honeypots no Curto Prazo
}

\author{
Gustavo José Neves da Silva \\ gustavo.neves@yandex.com \\ Universidade do Estado de Santa Catarina - UDESC \\ Joinville, Santa Catarina
}

\begin{abstract}
Honeypots are computing resources whose value lies in being probed, attacked, or compromised. They are security tools instrumental in observing attackers' behavior and understanding attack dynamics. Honeypots generate a large amount of monitoring data, which are often processed and analyzed manually. This introduces a delay in the perception of atypical occurrences and changes in trends, often rendering a deeper analysis of such phenomena unfeasible. We introduce a platform for the periodic automated processing of data collected by honeypots. The platform generates statistics and graphs from a set of traffic capture files in the PCAP format, and its modular design allows it to be easily extended and adapted to produce different outputs.
\end{abstract}

\section{KEYWORDS}

Honeypots, Segurança computacional, Redes de computadores

\section{INTRODUÇÃO}

Um honeypot é um recurso computacional de segurança dedicado a ser sondado, atacado ou comprometido [1]. O monitoramento do tráfego de um honeypot permite a observação de diversos tipos de comportamento malicioso, tais como ataques. Como resultado do monitoramento, honeypots podem produzir grandes volumes de dados, que muitas vezes são processados e analisados manualmente. Isso pode fazer com que anomalias e mudanças de tendências no tráfego sejam percebidas muito tempo após terem acontecido, impossibilitando uma investigação mais aprofundada sobre essas ocorrências e suas causas.

Diversas descrições de arquiteturas de honeypots podem ser encontradas na literatura [1-8]. Em [6, 8] são mencionadas algumas ferramentas de análise de dados produzidos por honeypots, mas as ferramentas citadas não estão disponíveis publicamente. Ainda, em [6] é proposto um formato para representação e troca de dados coletados por honeypots. No entanto, as referências encontradas não detalham o processo de geração de estatísticas a partir dos dados coletados pelos honeypots.

\section{SOLUÇÃO PROPOSTA}

O presente trabalho tem como objetivo propor uma plataforma para o processamento automatizado diário dos dados coletados por honeypots. Essa plataforma será usada no processamento de dados do HReflector [9], um honeypot para a observação de ataques distribuídos de negação de serviço por reflexão (DRDoS, distributed reflection denial of service). Ataques DRDoS exploram protocolos requisição-resposta com características de amplificação (que podem gerar respostas muito maiores que requisições), e utilizam endereços IP de origem forjados para que as respostas sejam redirecionados para o alvo do ataque [10]. O HReflector usa o Tcpdump [11] para

\author{
Rafael R. Obelheiro \\ rafael.obelheiro@udesc.br \\ Universidade do Estado de Santa Catarina - UDESC \\ Joinville, Santa Catarina
}

captura de tráfego, e armazena os dados no formato PCAP, o qual é comumente usado em honeypots [6]. Para evitar a manipulação de arquivos muito grandes, o próprio Tcpdump separa os arquivos em chunks de $100 \mathrm{MB}$; com isso, o tráfego de um dia pode estar disperso por vários arquivos dentro de um mesmo diretório.

O fluxo de processamento da plataforma proposta é mostrado na Figura 1. No passo 1, determina-se quais arquivos PCAP contêm o tráfego do dia de interesse, usando a ferramenta capinfos [12]. No passo 2, esses arquivos são combinados em um único arquivo usando a ferramenta mergecap [12]. No passo 3, os dados de pacotes contidos no arquivo PCAP são agregados em fluxos (flows) [13], ou seja, dados de sessões em intervalos de 5 minutos, usando a ferramenta argus [14]. No passo 4, o arquivo de fluxos é convertido para um arquivo de texto puro usando a ferramenta ra [14]. No passo 5, o arquivo gerado no passo 4 é processado para obter tabelas e gráficos com estatísticas de tráfego por ASN (5a), protocolo (5b) e país (5c), usando o pacote estatístico R [15]. Todo o fluxo de processamento é orquestrado por um script $\mathrm{R}$.

A Figura 2 mostra uma tabela e um gráfico gerados pela ferramenta, com estatísticas do tráfego diário por país. Como os dados referem-se a ataques DRDoS, as vítimas de ataques são caracterizadas pelos endereços IP de origem dos pacotes, devido ao uso de IP spoofing. Pode-se observar nesse exemplo que, no dia 29/09/2018, a maior parte das vítimas de ataques DRDoS estava situada nos Estados Unidos (US), com o total de 32,61 MB, e que o pico de tráfego para esse país foi de $1,72 \mathrm{~KB} / \mathrm{s}$. O ataque mais intenso (pico mais à direita no gráfico) ocorreu às 22:35, e teve vítimas situadas na China, com intensidade de $22,29 \mathrm{~KB} / \mathrm{s}$. De acordo com a Figura 3, essas vítimas estavam concentradas no sistema autônomo identificado pelo ASN 4134.

\section{CONSIDERAÇÕES FINAIS}

O processamento automatizado de dados gerados por honeypots é essencial para permitir o acompanhamento contínuo dos ataques observados por essas ferramentas. Com esse acompanhamento é possível identificar anomalias e mudanças de tendências no tráfego pouco tempo depois do sua ocorrência, e consequentemente aprofundar a investigação sobre suas causas, tanto pela ampliação da instrumentação dos honeypots quanto pelo acesso a dados de monitoramento mais voláteis que ajudem a elucidar os fenômenos. Este trabalho apresenta uma plataforma que facilita o processamento periódico de dados armazenados no formato PCAP. Embora desenvolvida com foco no HReflector, a plataforma pode ser facilmente adaptada a outros honeypots que armazenem dados nos formatos PCAP ou de fluxos. Na continuidade desta pesquisa, pretende-se colocar a plataforma em produção e disponibilizar seu código fonte sob uma licença livre. 


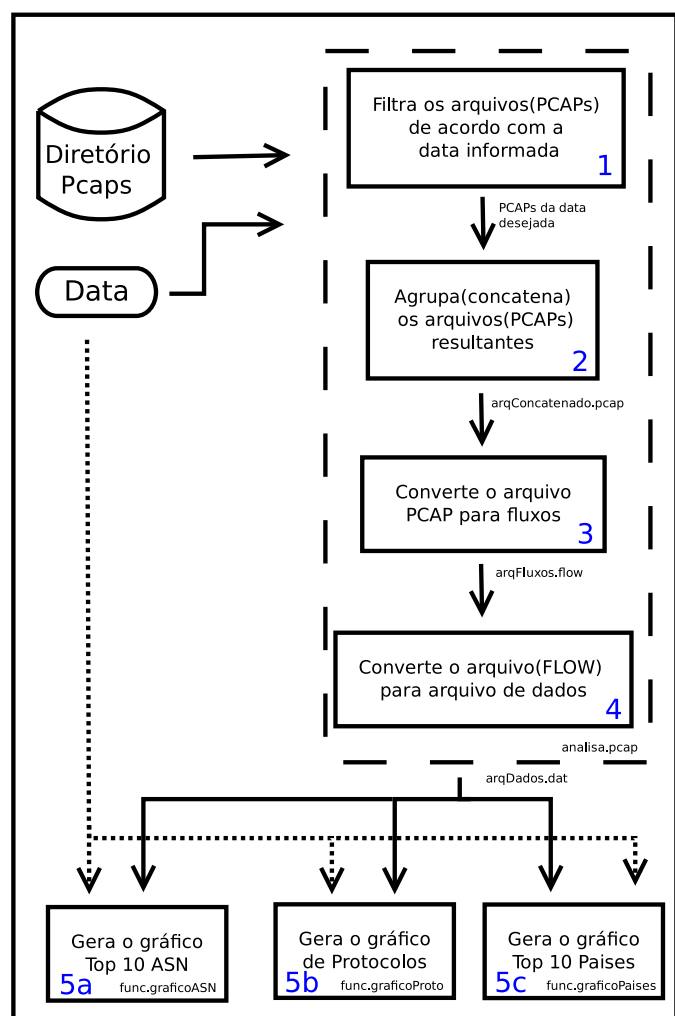

Figura 1: Fluxo de processamento da plataforma proposta.

\section{AGRADECIMENTOS}

Os autores agradecem o apoio da UDESC e da FAPESC para a realização desta pesquisa. Gustavo Neves da Silva foi bolsista PRO$\mathrm{BIC} / \mathrm{UDESC}$.

\section{REFERÊNCIAS}

[1] Lance Spitzner. Honeypots: Tracking Hackers. Addison-Wesley Professional, 2002. ISBN $9780321108951,0321108957$.

[2] Eric Alata, Vincent Nicomette, Mohamed Kaâniche, Marc Dacier, and Matthieu Herrb. Lessons learned from the deployment of a high-interaction honeypot. In 2006 Sixth European Dependable Computing Conference, pages 39-46. IEEE, 2006

[3] Cristine Hoepers, Klaus Steding-Jessen, Luiz ER Cordeiro, and Marcelo HPC Chaves. A national early warning capability based on a network of distributed honeypots. In 17th Annual FIRST Conference on Computer Security Incident Handling, Singapore, pages 2-5, 2005.

[4] Eric Alata, Marc Dacier, Yves Deswarte, M Kaaâniche, Kostya Kortchinsky, Vincent Nicomette, Van-Hau Pham, and Fabien Pouget. Collection and analysis of attack data based on honeypots deployed on the internet. In Quality of Protection, pages 79-91. Springer, 2006

[5] David Watson and Jamie Riden. The honeynet project: Data collection tools, infrastructure, archives and analysis. In 2008 WOMBAT Workshop on Information Security Threats Data Collection and Sharing, pages 24-30. IEEE, 2008.

[6] Cristine Hoepers, Nandamudi L Vijaykumar, and Antonio Montes. Hidef: a data exchange format for information collected in honeypots and honeynets. INFOCOMP, 7(1):86-95, 2008.

[7] Peter Pisarčík and Pavol Sokol. Framework for distributed virtual honeynets. In Proceedings of the 7th International Conference on Security of Information and Networks, page 324. ACM, 2014.

[8] Marcin Nawrocki, Matthias Wählisch, Thomas C. Schmidt, Christian Keil, and Jochen Schönfelder. A survey on honeypot software and data analysis. CoRR, abs/1608.06249, 2016. URL http://arxiv.org/abs/1608.06249.
[9] Tiago Heinrich and Rafael R. Obelheiro. Brasil vs mundo: Uma análise comparativa de ataques DDoS por reflexão. In XIX Simpósio Brasileiro de Segurança da Informação e de Sistemas Computacionais (SBSeg), São Paulo, 2019.

[10] Cert. Recomendações para melhorar o cenário de ataques distribuídos de negação de serviço (ddos). URL https://www.cert.br/docs/whitepapers/ddos/.

[11] The Tcpdump team. Tcpdump/libpcap public repository. URL http://www. tcpdump.org/.

[12] Wireshark Foundation. Wireshark. URL https://www.wireshark.org/.

[13] Richard Bejtlich. The Practice of Network Security Monitoring. No Starch Press, 2013.

[14] Qosient. Argus - auditing network activiy. URL https://www.qosient.com/argus/.

[15] R Core Team. R: A Language and Environment for Statistical Computing. R Foundation for Statistical Computing, Vienna, Austria, 2019. URL https://www.Rproject.org/. 
XI Computer on the Beach

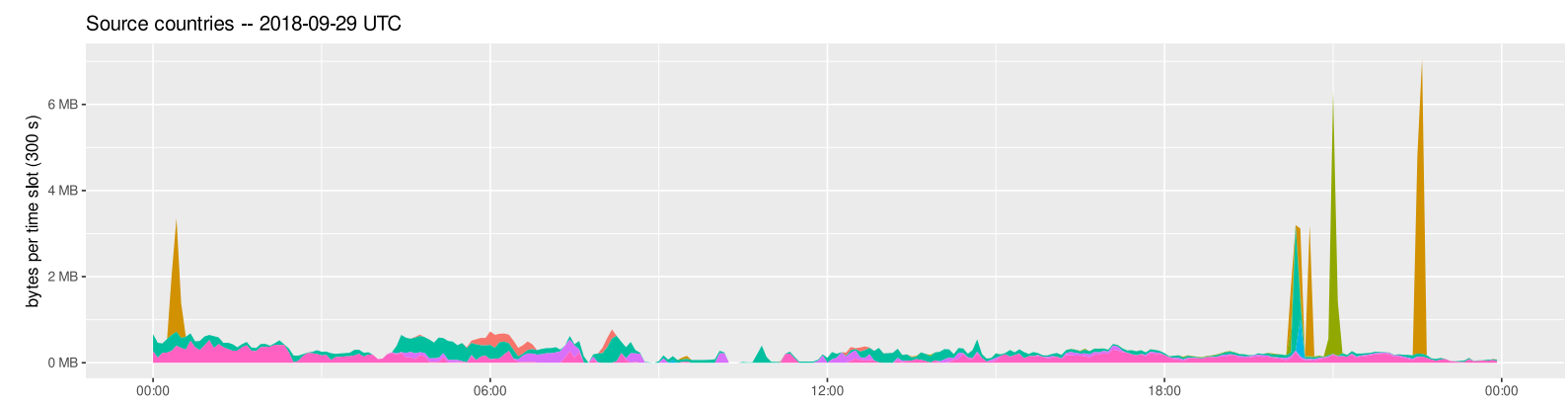

\begin{tabular}{|c|c|c|c|c|}
\hline Country_code & Country_code & Total & Max & Avg \\
\hline US & United States & 32.61 MB $29.89 \%$ & $1.72 \mathrm{~KB} / \mathrm{s}$ & $0.39 \mathrm{~KB} / \mathrm{s}$ \\
\hline $\mathrm{CN}$ & China & 20.98 MB $19.23 \%$ & $22.30 \mathrm{~KB} / \mathrm{s}$ & $0.25 \mathrm{~KB} / \mathrm{s}$ \\
\hline GB & United Kingdom & $9.18 \mathrm{MB} 8.42 \%$ & $0.91 \mathrm{~KB} / \mathrm{s}$ & $0.11 \mathrm{~KB} / \mathrm{s}$ \\
\hline FR & France & 8.56 MB $7.84 \%$ & $19.86 \mathrm{~KB} / \mathrm{s}$ & $0.10 \mathrm{~KB} / \mathrm{s}$ \\
\hline$A U$ & Australia & $3.50 \mathrm{MB} 3.21 \%$ & $1.02 \mathrm{~KB} / \mathrm{s}$ & $0.04 \mathrm{~KB} / \mathrm{s}$ \\
\hline RU & Russia & 0.88 MB $0.81 \%$ & $2.80 \mathrm{~KB} / \mathrm{s}$ & $0.01 \mathrm{~KB} / \mathrm{s}$ \\
\hline IE & Ireland & $0.03 \mathrm{MB} \quad 0.02 \%$ & $0.04 \mathrm{~KB} / \mathrm{s}$ & $0.00 \mathrm{~KB} / \mathrm{s}$ \\
\hline TW & Taiwan & $0.01 \mathrm{MB} \quad 0.01 \%$ & $0.03 \mathrm{~KB} / \mathrm{s}$ & $0.00 \mathrm{~KB} / \mathrm{s}$ \\
\hline & Others & 33.35 MB $30.57 \%$ & $9.41 \mathrm{~KB} / \mathrm{s}$ & $0.40 \mathrm{~KB} / \mathrm{s}$ \\
\hline
\end{tabular}

Figura 2: Estatísticas diárias de tráfego por país.

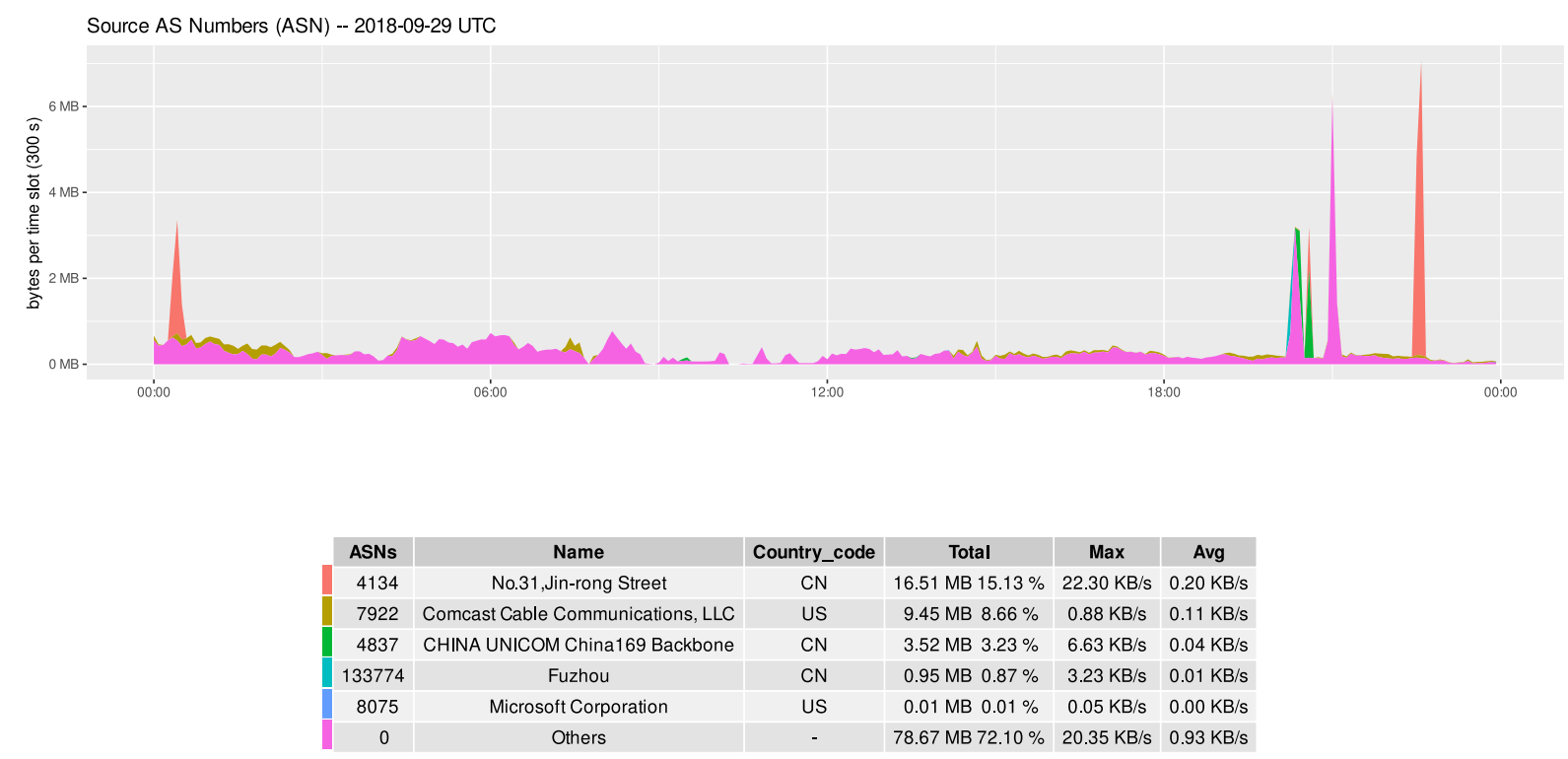

Figura 3: Estatísticas diárias de tráfego por ASN. 